\title{
Effectiveness of hearing conservation program at a large surface gold mining company in Ghana.
}

\author{
Geoffrey Kwabla Amedofu \\ Department of Eye, Ear, Nose and Throat, School of Medical Science, Kwame Nkrumah University of \\ Science and Technology, Kumasi, Ghana, West Africa.
}

\section{SUMMARY}

\begin{abstract}
A study conducted to determine the effectiveness of a Hearing Conservation Programme (HcP) was conducted in a surface gold mining Company in Ghana. The procedure adopted included a retrospective review and comparison of individual Audiograms from 1999-2003. The analysis of data was based on 200 workers at various departments namely Pit, processing, geology, survey, engineering, utility Health and Safety. The criteria used was an average shift of $10 \mathrm{~dB}$ or more at 2000 , 3000 and $4000 \mathrm{~Hz}$. The result showed that $7 \%$ developed a positive and negative hearing loss. That is, hearing loss got worse in $5.5 \%$ while it has actually improved in $1.5 \%$ of the workers. Again, 45 workers had shifts of less than $10 \mathrm{~dB}$ at frequencies, 500, 1000, 2000 and $4000 \mathrm{~Hz}$. Out of this $(45), 27 \%$ had shifts of less than $10 \mathrm{~dB}$ at frequencies not included in the criteria. The Hearing Conservation Programme (HCP) is effective since less than $6 \%$ of workers have additional hearing loss.
\end{abstract}

[Afr J Health Sci. 2007; 14:49-53]

\section{Introduction}

Hearing loss caused by noise (Noise-Induced Hearing Loss i.e. NIHL) is one of the most common hazards in both industrialized and developing countries. Hazardous noise exposure and the resultant NIHL are considered the most complex and far-reaching problems in the health and safety employment.

In Ghana, the overwhelming liability for hazardous noise and NIHL is that imposed by the Factories, offices and shops Act [1]. Although excessive noise exposure has been recognised as harmful to the ears, very little attention has been paid to reduce noise at the source to prevent it's transmission from the source to the workers. The generally acceptable standard regulation in most countries including Ghana is that a noise level of $85 \mathrm{~dB}$ for an $8-$ hour daily exposure is potentially damaging [2]. In several developed countries, awareness and acceptance of the reality of NIHL as an occupational hazard has increased. As a result of this, there is a gradual change in focus from recognising and treating hearing loss to preventing it through effective hearing conservation programmes [3].

In developed countries, employers in factories and mining companies are required by law to develop HCPs to protect workers against hazardous noise exposure. In Ghana, the potential danger of NIHL has been recognised
[4]. Thus, the only logical, practical and long term approach to prevent NIHL is for mining companies to develop HCPs to protect workers exposed to hazardous noise at the work place. HCP refers to an organised activity aimed at prevention of NIHL, typically including noise measurements, routine Audiometry, noise reduction and education of workers primarily in the use of hearing protectors.

In 1999, a large surface gold mining company in Ghana embarked on a HCP to protect the hearing of its workers. Attaining the goals of HCPs is, however, difficult, if not impossible without a systematic method of judging the effectiveness of HCP. In order to judge the effectiveness of a particular HCP in reducing on-the-job noise exposure and subsequent hearing loss resulting from such exposure, some quantifiable measure must be available. The obvious choice is the audiogram. The choice of the audiogram as the appropriate measure of the effectiveness of the HCP is not meant to imply that audiometric tests are free from artifacts. It simply suggests that results of hearing tests are more related to the effectiveness of the overall HCP than other parts of the program. Various indicators of HCPs have been proposed [5]. 


\section{Potential early Indicators: Variability Indices}

The American National Standards Institute (ANSI) has released standard protocols for judging the effectiveness of hearing conservation programs [6]. These protocols measure year-toyear audiometric variability, suggesting that excessive audiometric variability may identify either inadequate audiometric testing procedures or inadequate hearing protector use in hearing conservation programs. If poor quality audiometric testing can be ruled out as a contributor to excessive audiometric variability, then audiometric data are thought to be contaminated by temporary threshold shift (TTS), thus questioning the effectiveness of HCPs. The three protocols recommend by ANSI are as follows:

1. Percent worse sequential (\%WS): In this case, the percentage of audiograms demonstrating a $15 \mathrm{~dB}$ change for the worse at any test frequency $(500-6000 \mathrm{~Hz})$ in the either ear was used.

2. $\quad$ Percent better or worse sequential (\%BWS): The percent of audiograms with $15 \mathrm{~dB}$ changes for the better or worse at any test frequency $(500-6000 \mathrm{~Hz})$ in either ear.

3. Standard deviation of threshold shifts: Computed from the binaurally averaged audiometric frequencies at $0.5,1,2,3,4$, and $6000 \mathrm{~Hz}$ and grouped frequency combinations of $0.5-3 \mathrm{KHz}, 2-4 \mathrm{KHz}$ and $3-6 \mathrm{KHz}$ criterion ranges of acceptable, marginal, and unacceptable levels of audiometric variability are published in the draft standard for trial use, comment and criticism [6].

\section{Shift Indices \\ Occupational safety and Health Administration (OSHA) standard Threshold Shift:}

Noise regulations promulgated by the Occupational Safety and Health Administration [7] in the United States of America, specify that baseline and annual audiograms be obtained from employees who are occupationally exposed to noise at or above $85 \mathrm{~dB}$ (A) for a timeweighted average of 8 hours. Each annual audiogram is compared to baseline audiograms (i.e. initial audiograms) to monitor potential changes in hearing level due to noise exposure. An average audiometric change in either ear of $10 \mathrm{~dB}$ or greater at 2,3 and $4 \mathrm{KHz}$ is defined as the standard threshold shift (STS). In the USA, regulatory pressures cause many HCPs to track
OSHA STS rates for compliance purposes. Consequently the use of OSHA STS rates for assessing performance is fairly widespread. In many African countries, there are laws to protect workers from hazardous noise. However, inconsistent compliance, spotty enforcement of governmental regulation and lack of comprehensive hearing conservation programs have been the underlying cause of noise-induced hearing loss in these countries. In 1999, a large surface gold mining company embarked on a hearing conservation program to protect its workers from hazardous noise. The HCP embarked upon included noise measurements, routine Audiometry, display of posters and warning signals at hazardous noise areas in the company to alert workers use of hearing protectors and education primarily in the use of hearing protectors. The purpose of this investigation was to examine the first audiogram (baseline) obtained in 1999 and the $5^{\text {th }}$ Audiogram obtained in 2003. Another rationale was to determine whether the HCP embarked on was effective. A secondary objective was to examine the efficacy of OSHA STS for early detection of unacceptable HCP performance. The study was based on the assumption that the baseline audiogram and subsequent audiograms are sufficient enough to estimate $\mathrm{HCP}$ effectiveness.

\section{Materials and Methods}

Audiometric data compiled at a large surface Gold Mining Company were examined retrospectively as a means of evaluating the hearing conservation program. Data consists of audiograms of 200 w0rkers at various departments in the company namely pit processing, geology, mining, survey, safety Health and environments. At the start of the HCP in 1999, individual work histories were collected and noise levels in different working conditions were measured. Those who were exposed to free time and military noise were not included in the data. Thus, in all, 200 baseline audiograms obtained in 1999 were compared with 200 audiograms obtained from the same workers in 2003 [6]. Shifts of $10 \mathrm{~dB}$ or greater in either ear at 2, 3 and $4 \mathrm{KHz}$ were classified as STS. In order to determine the effectiveness of the HCP the percent of employees exceeding the STS criterion in a positive direction only was used, and for this measure, a less than $6-8 \%$ should show additional hearing loss in an effective program [8]. Only hearing level 
declines were considered and age correction factors were not employed in calculation of these shifts.

\section{Results}

The primary goal of HCP is the elimination of noise induced permanent threshold shift resulting from on the job noise exposure. Out of the two hundred audiograms, which were examined, 11 $(5.5 \%)$ showed an increase in thresholds of more than $10 \mathrm{~dB}$ (i.e. indicating worsening hearing levels) while $3(1.5 \%)$ showed a decrease in thresholds (i.e. hearing levels showed an improvement). Table I depicts the individual data of workers who showed $\mathrm{a}>10 \mathrm{~dB}$ change in hearing sensitivity.

Table 1: Workers who developed 10dB or more change hearing loss at 2000,3000 and $4000 \mathrm{~Hz}$ (mean values) in one or both ears.

\begin{tabular}{|c|c|c|c|c|}
\hline & Age & $\begin{array}{l}\text { Rate of Change (dB) } \\
\text { Better }\end{array}$ & Worse & Department \\
\hline $\mathrm{X}_{1}$ & 45 & - & 20 & Geology \\
\hline $\mathrm{X}_{2}$ & 30 & 12 & - & Geology \\
\hline $\mathrm{X}_{3}$ & 38 & - & 10 & Utility \\
\hline $\mathrm{X}_{4}$ & 54 & 12 & - & Engineering \\
\hline $\mathrm{X}_{5}$ & 48 & - & 20 & SSD \\
\hline $\mathrm{X}_{6}$ & 49 & 10 & - & Processing \\
\hline $\mathrm{X}_{7}$ & 44 & - & 10 & Processing \\
\hline $\mathrm{X}_{8}$ & 39 & - & 10 & Processing \\
\hline $\mathrm{X}_{9}$ & 41 & - & 10 & Processing \\
\hline $\mathrm{X}_{10}$ & 36 & - & 20 & Processing \\
\hline $\mathrm{X}_{11}$ & 37 & - & 10 & Geology \\
\hline $\mathrm{X}_{12}$ & 43 & - & 12 & Cook/Kitchen \\
\hline $\mathrm{X}_{13}$ & 28 & - & 20 & Processing \\
\hline $\mathrm{X}_{14}$ & 32 & - & 12 & Geology \\
\hline
\end{tabular}

We also see figure I data from 45 employees using less than $10 \mathrm{~dB}$ shift criterion $(<10 \mathrm{~dB})$ at any frequency to determine degree of variability in the data. As indicated in the figure, the largest number of $\leq 10 \mathrm{~dB}$ shifts occurred at $4000 \mathrm{~Hz}$
$(50 \%)$, followed by $2000 \mathrm{~Hz}, 1000 \mathrm{~Hz}$ and $500 \mathrm{~Hz}$. Another important point with regard to this finding is that about $27 \%$ shifts occurred at frequencies not included in the criterion recommended by OSHA [8]. 
Figure 1: Employees showing threshold shifts of $<10 \mathrm{~dB}$ as a function of frequency $(500,1000,2000$ and $4000 \mathrm{~Hz}$ ).

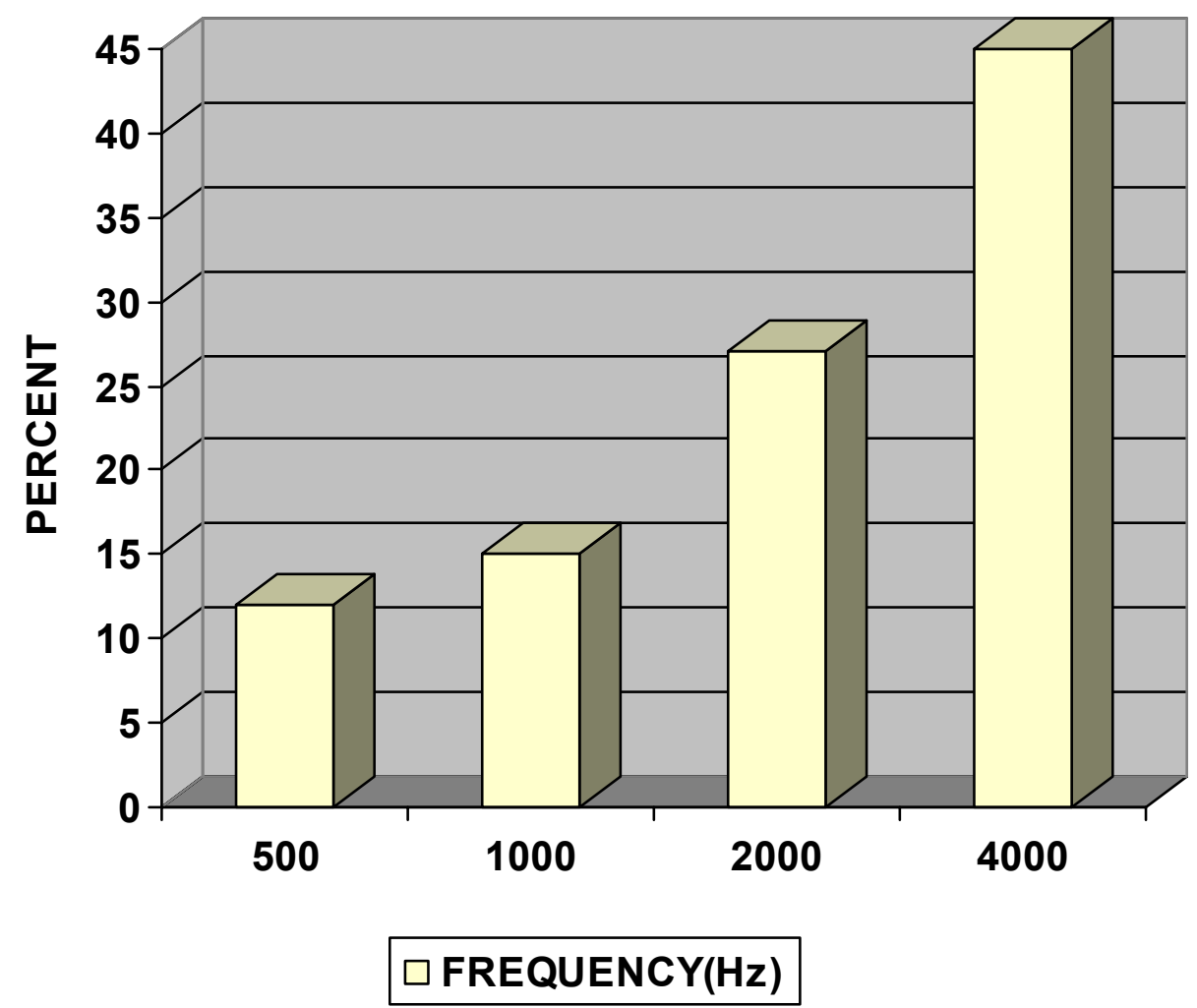

\section{Discussion}

The purpose of HCPs is the elimination of noiseinduced hearing loss. The present data has shown that $14(7 \%)$ out of 200 workers developed a positive and negative change in hearing using a $10 \mathrm{~dB}$ or more criteria at 2000,3000 and $4000 \mathrm{~Hz}$. That is, hearing threshold levels have actually improved (better thresholds) in $1.5 \%$ of the workers, while in $5.5 \%$ the hearing levels got worse. A simple method recommended for determining the effectiveness of HCPs is the percent of employees exceeding STS criterion in a positive direction only (worsening threshold levels). And for this measure, a less than 6- 8\% should show additional hearing loss in an effective programme [8]. Seen thus, it is reasonable to surmise that the HCP in this mining company is effective since less than $6 \%$ $(5.5 \%)$ of the workers have additional hearing loss. Some HCPs include data base analysis programmes, which assess data on the noise immission level, including the evaluation of factors other than workplace noise to predict Noise-Induced Hearing Loss (NIHL) from occupational noise exposure [9]. This computerbased analysis was not utilised in this analysis.

It was reported that 45 employees showed less than $10 \mathrm{~dB}$ shift across frequencies from 500 through $4000 \mathrm{~Hz}$. Out of this, $27 \%$ of shifts occurred at frequencies not included in the criterion recommended by OSHA. Melnick [10] proposed that in clinical Audiometry a $10 \mathrm{~dB}$ hearing shift at any frequency is important but in screening Audiometry a $15 \mathrm{~dB}$ HL shift should be considered significant. Therefore the less than $10 \mathrm{~dB}$, which were observed at the frequencies from $500 \mathrm{~Hz}$ through $4000 \mathrm{~Hz}$, are insignificant. Again, it would appear that HCPs based on noise exposure alone are not adequate in preventing STS. There is the argument that the effect of noise and aging alone might not account for the STS that we see at the workplace. Fundamental to this notion is the report that the effect of noise in the workplace can be exacerbated by temperature, vibration and chemicals [11]. The fact is pure-tone audiometric thresholds only identify the magnitude of the hearing loss not the etiology. The audiometric configuration i.e. in cases of noise-induced hearing loss and ototoxity can be identical [12]. If careful analysis of these 
results were not performed and attention given to all the exposure conditions, it is conceivable that the observed hearing disorders were erroneously solely attributed to noise. Ultimately, an increase in the awareness of ototoxic potential of chemicals should contribute to the improvement of preventive strategies that can help reduce the risk of work-related hearing loss.

\section{Conclusion}

In 1999, a large surface gold mining company has embarked on a HCP to protect the hearing of its workers'. In order to examine the effectiveness of this programme, the OSHA STS was used. That is, an average Audiometric change in either ear of $10 \mathrm{~dB}$ or more at 2, 3 and $4 \mathrm{KHz}$ defined as the standard threshold shift was employed. We observed that $5.5 \%$ of the workers whose audiograms were reviewed exceeded the STS criterion in a positive direction. According to Royster and Royster, in an effective program less than 6-8\% should show additional hearing loss. Therefore, the HCP in this company was effective. The management of this company has to be commended for protecting its workers. The occupational physician at the company actively participated in all aspects of the HCPs through ongoing evaluation of programme outcomes and processes. Unfortunately, many employers are not interested in HCPs in Ghana, due to the cost involved. Future attempts to identify valid indicators of HCP effectiveness in this company should consider both variability outcomes and shift criteria such as ANSI \%Ws and OSHA STS. Both ANSI \%Ws and STS outcomes directly reflect hearing protector program integrity and indirectly reflect audiometric testing program integrity.

\section{References}

1. Factories, shops and offices Act in Ghana. 1970; p: 1-3.

2. NIH Consensus Development Conference. Consensus Statement. January $23^{\text {rd }}-24^{\text {th. }} 1990 ; 3,1-21$.

3. Suiter TC, Franks JR and Dunn JR, $\underline{A}$ practical guide to effective hearing conservation programmes in the workplace. National Institute for Occupational Safety and Health, 1990; p90-122.

4. Health and safety in Mining Companies - Report on Workshop, Ministry of Mines and energy, 1999; p2-15.
5. Simpson TH, Stewart $M$ and Kaltenbach JA. Early indicators of Hearing conservation programme Performance. Journal of American Academy of Audio logy. 1994; 5:300-6.

6. American National Standards Institute. Draft American National Standards: Evaluating the Effectiveness of Hearing Conservation Programmes. (ANSI S12. 13 - 1991, New York ANSI.

7. Occupational safety and Health Administration. Occupational Noise Exposure, Hearing Conservation Amendment: Final Rule: Federal (USA) Register, 1983; 46:9785.

8. Royster LH, Royster JD and Bevger JD Methods of evaluating hearing conservation database. In: Alberti PW (Ed.) Personal Hearing protection in Industry._New York Raven Press 1982.

9. Franks JR, Davis RR and Kreig EF jr. Analysis of Hearing Conservation Programme database: Factors other than workplace noise. Ear and Hearing. 1989; 10:273-80.

10. Melnick W. Evaluation of Hearing Conservation programmes: A review and analysis. American Industrial Hygiene Association Journal. 1984; 45: 459-67.

11. Morata TC, Franks JR and Dunn DE. Unmet needs in Occupational hearing conservation. Lancet. 1994; 344:479

12. Morata TC. Interaction between Noise and Asphyxiants. A concern for Toxicology and Occupational Health. Toxicological Sciences. 2002; 66:1-3. 